\title{
Detection and Localization of Parathyroid Adenoma and its Consequences in a Patient with Primary Hyperparathyroidism
}

\section{Luljeta Abdullahu $^{1 *}$ and Naser Gjonbalaj ${ }^{2}$}

${ }^{1}$ Department of Nuclear Medicine, University Clinical Center of Kosova, Republic of Kosovo

${ }^{2}$ Clinic of Radiology, Prishtina Republic of Kosova, University Clinical Center of Kosova, Republic of Kosovo

*Corresponding Author: Luljeta Abdullahu, Department of Nuclear Medicine, University Clinical Center of Kosova, Republic of Kosovo.
Received: July 26, 2021

Published: August 24, 2021

(C) All rights are reserved by Luljeta

Abdullahu and Naser Gjonbalaj.

\begin{abstract}
Purpose: The purpose of this presentation was in addition to assessing the finding of parathyroid adenoma by parathyroid scan, the use of additional methods where we also used the moment to perform SPECT Rest non gatted and myocardial perfusion with the same radionuclide 99mTc-MIBI and taken as thyroid and parathyroid ultrasonography, DEXA, and 99mTc DTPA dynamic renal scintigraphy.

Case Report: The 59-year-old patient presented for parathyroid gland scintigraphy at the Nuclear Medicine Service. Comes with a dg. HTA, Nephrocalculosis. CKD 4, with high values of PTH (Parathormone) $2297 \mathrm{pg} / \mathrm{mil}$. and with a history of almost 20 years which suffered from kidney stones (Nephrocalculosis). Suffers from high blood pressure for about 8 years, treated with Lorista $\mathrm{H}$, Bipreso and Aspirin.

Method: Ultrasound of the thyroid and parathyroid gland was performed with a $7 \mathrm{~Hz}$ probe. In the parathyroid image at the time of application of the diagnostic dose of $20 \mathrm{mCi}$ of 99mTc-MIBI 2-minute frames were taken for 20 min using zoom 4.0 and $128 \mathrm{x} 128$ matrix with E-Cam Siemens gamma camera. At 50 - 60 minutes after i.v. delivery of 99mTc-Mibi, myocardial perfusion scintigraphy was performed with the one-day protocol only in rest without gated. In the following days, DEXA and kidney scan were performed

Results: Ultrasonographic findings (in the distal part of the right lobe, an anecogenic to slightly hypoechoic nodule formation with increased peripheral vascularization was found. A positive MIBI scan for parathyroid adenoma was identified as an area of increased focal intake in the distal part of right lobe. On myocardial perfusion scintigraphy we found hypertrophy of the septal and lateral wall of the LV. In DEXA severe osteoporosis as well as in the kidney scan hypofunction of both kidneys. 30.04.2021. St Post op. Dg. HP. Adenoma Glandulae Parathyroideae lat dex.
\end{abstract}

Keywords: Adenoma Parathyroides; 99m-Tc-MIBI; Ecam Gamma Camera; Ultrasonography; Myocadial Perfussion Imagin (MPI); DEXA; Renal Scan 99mTc-DTPA

\section{Introduction}

The secretion of parathyroid hormone (PTH), by two pairs of parathyroid glands, located in the back of the thyroid gland, regulate serum calcium concentration and bone metabolism. PTH pro- motes the release of calcium from the bones, increases the absorption of calcium from the intestine and increases the absorption of calcium in the renal tubules. In turn, serum calcium concentration regulates PTH secretion, a mechanism mediated through a calcium-sensing receptors on the surface of parathyroid cells. 
Primary pHPT hyperparathyroidism is caused by the secretion of excessive amounts of PTH by one or more enlarged parathyroid glands. Patients with pHPT may suffer from kidney stones, osteoporosis, gastrointestinal symptoms, cardiovascular disease, muscle weakness and fatigue, and neuropsychological disorders. The highest prevalence of the disease is found in postmenopausal women.

A prevalence of $2 \%$ was found from screening in postmenopausal women [1].

Parathyroid adenomas (PTAs) are usually small, $<2 \mathrm{~cm}$ in size and weighing < $1 \mathrm{gm}$ ]. Giant PTA (GPTA), although rare, is commonly defined as weighing $>3.5 \mathrm{gm}$, with some reports describing weights up to $110 \mathrm{~g}$.

\section{Laboratory analysis}

From laboratory tests Parathyroid adenomas are usually detected when a higher than normal level of calcium appears in a routine blood test, especially in asymptomatic people, and doctors are required to confirm the diagnosis of primary hyperparathyroidism with a test that shows that the levels of parathyroid hormone in the blood are higher than normal.

\section{Choice of imaging technique}

The most common imaging methods for the detection and localization of parathyroid adenomas are ultrasound and radionuclide scintigraphy as the two main methods [2].

99mTc-sestamibi scanning is now considered the most sensitive imaging technique in patients with pHPT [3].

Whatever protocol is used, $99 \mathrm{mTc}$-sestamibi scanning usually meets the ectopic gland detection requirement [4].

\section{9mTc-sestamibi}

The administered dose is usually 185 - $900 \mathrm{MBq}$; the typical dose is $740 \mathrm{MBq}$ This radiotherapy is localized to both sides of the parathyroid gland and functional thyroid tissue and is usually flushed out of normal thyroid tissue faster than from abnormal parathyroid tissue.

The exact mechanism of uptake remains unknown [5]. Taking 99mTc-sestamib depends on many factors, including perfusion, cell cycle phase and functional activity [6-11]. The final cellular localization of $99 \mathrm{mTc}$-sestamib is within the mitochondria. It accumulates in the mitochondria of many tissues but especially in normal cardiac and thyroid cells; is especially prominent in the parathyroid glands when they are overactive and held there preferentially [5].

\section{Case Presentation}

The 59-year-old patient presented for parathyroid gland scintigraphy at the Nuclear Medicine Service. Comes with a dg. HTA, Nephrocalculosis. CKD 4 nd, with high values of PTH (Parathormone) $2297 \mathrm{pg} / \mathrm{mil}$ and with a history of almost 20 years which suffered from kidney stones (Nephrocalculosis). Suffers from high blood pressure for about 8 years, treated with Lorista H, Bipreso and Aspirin.

The patient presents with these laboratory tests

Le 11; Hgb 11; HTC 33, PLT 33; urea 11, creatinine 205; glycemia 4.9; Alb 39; total protein 59.3, cholesterol 6.72. direct bilirubin 1.9; total bilirubin 6.9; Tg 1.72; uric acid 412; ALT 8; AST 12; Ph 7.40; PC2 33; At 142; K 4.1; $\mathrm{Ca}++2.24$ (high); $\mathrm{HCO}_{3} 20$; bECF-4.0.

From the imagery there were:. Kidney ultrasound with completely hyperechoic cortical parenchyma, with hyperechoic formations with intense dorsal shadow with $\mathrm{dm} 2-3 \mathrm{~mm}$, located mainly at the top of the calyxal pyramids without stasis.

\section{Working methods}

From the imaging methods by the radiologist and the nuclear doctor we used.

Ultrasound of the thyroid and parathyroid gland.

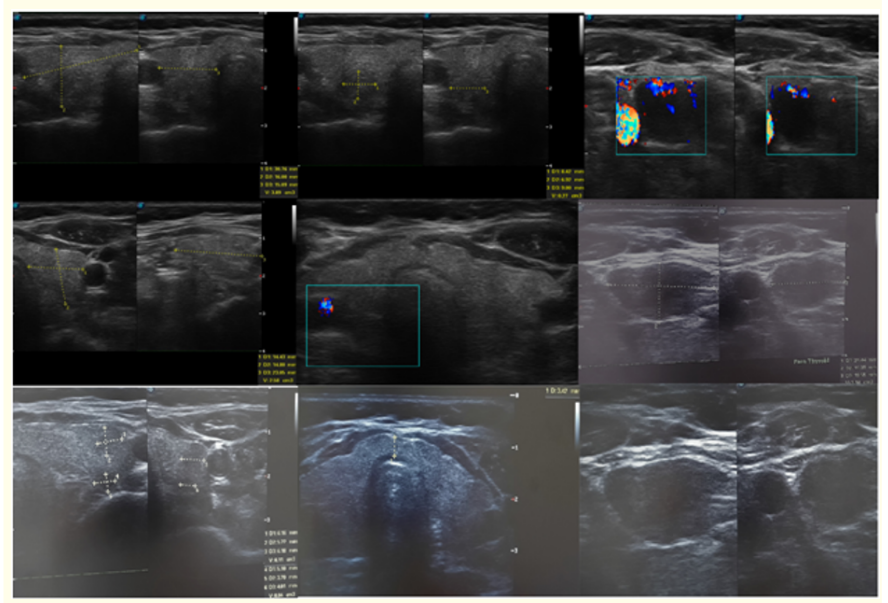

Image 1: Ultrasound thyroid and parathyroid. 
Ultrasound image shows a well-defined hypoechoic nodular lesion, in the right parotid gland (arrow).

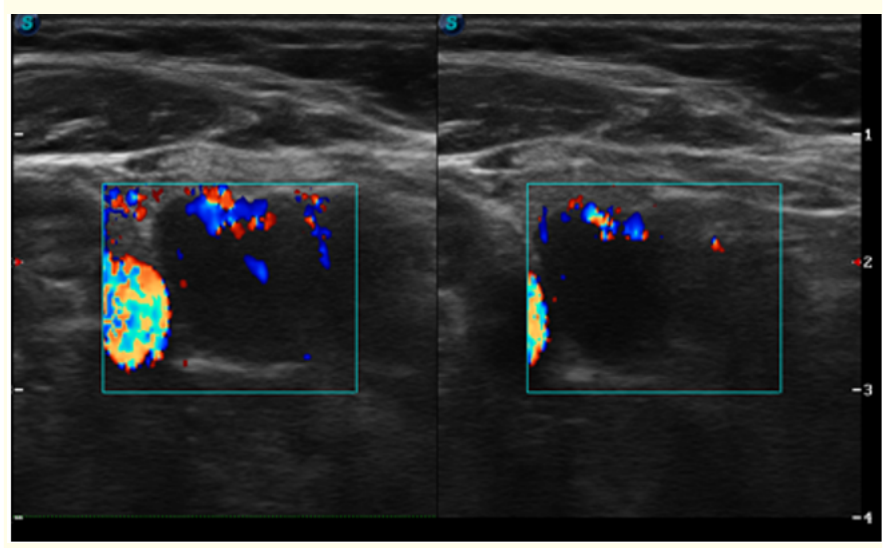

Image 2: Anecogenic to slightly hypoechoic nodule formation with increased peripheral vascularization was found.

Scanning with Technetium99m-sestamib for neck exploration in patients with primary hyperparathyroidism.

Parathyroid gland scintigraphy was performed on 26.03.2021 using early and late imaging after 99mTc-MIBI injection (twophase study).

A planar cervico-thoracic procedure (front view) was performed in the form of dynamic study, for $20 \mathrm{~min} 20$ sequences were obtained. Static images were taken in 20 minutes/anterior view and in 2-3 hours in both images after an intravenous injection of 20 $\mathrm{mCi}$ of MIBI (Image 3 and 4).

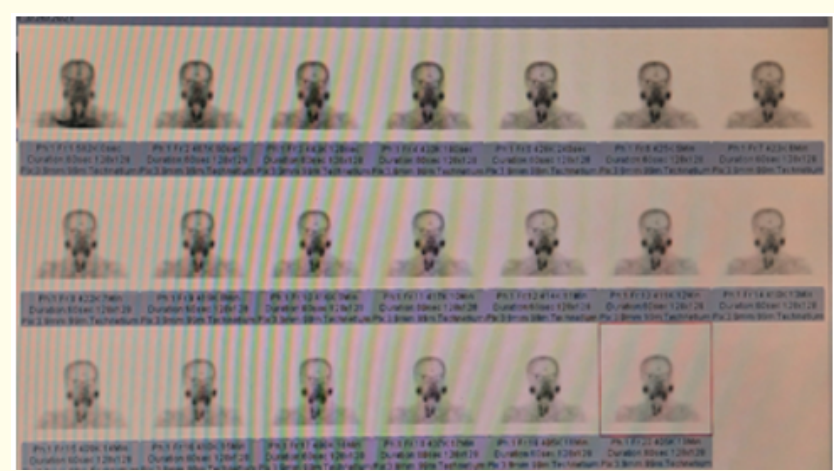

Image 3

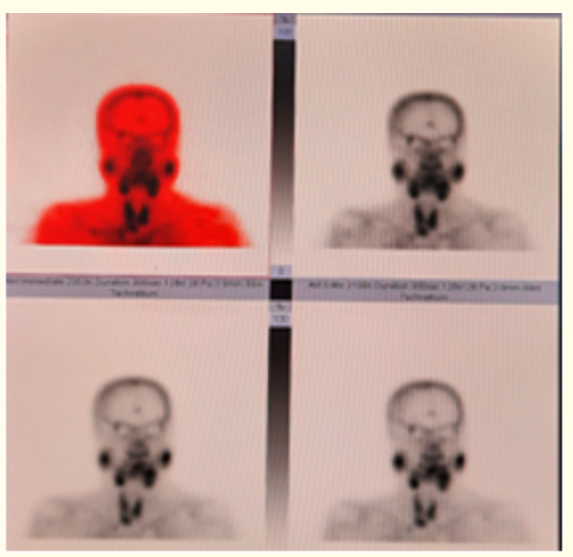

Image 4

A positive MIBI scan for parathyroid adenoma was defined as an area of increased focal uptake in the caudal part of the right lobe of the thyroid gland which continued in late imaging, as opposed to uptake into normal thyroid tissue which decreases progressively over time (differential rinsing), but at the same time we had intensive uptake (accumulation) even at the level of salivary glands (Image 5).

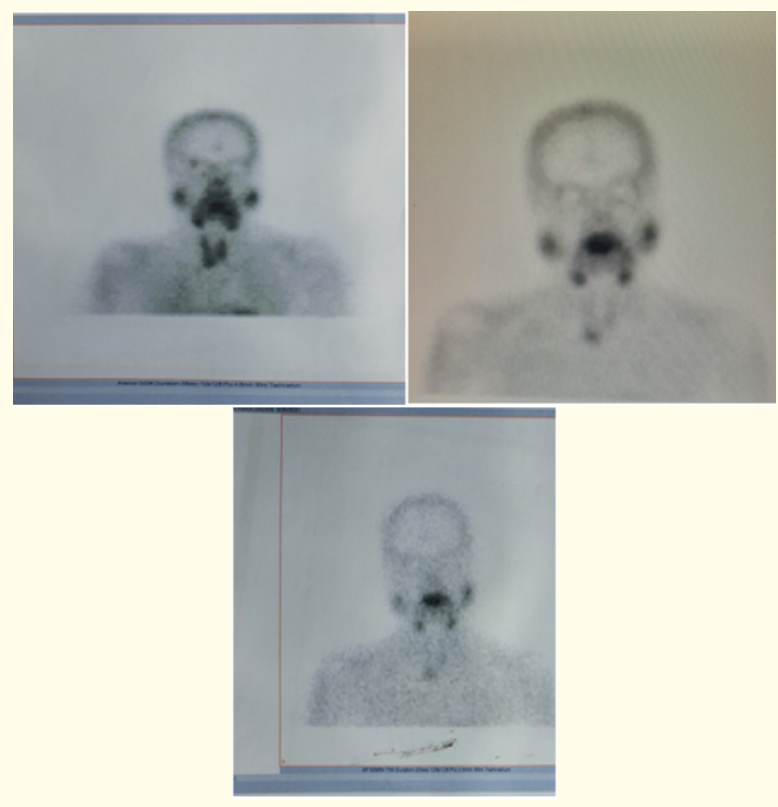

Image 5 
In order to use the same radiopharmaceutical, SPECT non gatted myocardial perfusion was performed, where we found good perfusion in all segments of the left ventricular muscle, with a hypertrophy of the lateral wall but more pronounced in the septal wall, with mild hypoperfusion in the inferior wall but without defects.

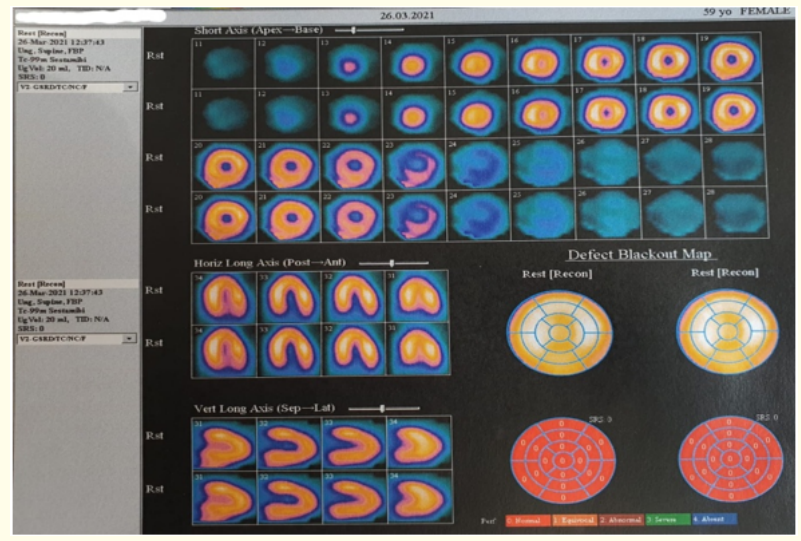

Image 6

In the following days, DEXA images were taken to assess the mineral density of the bones, wher e we found severe osteoporosis (Image 7 and 8).

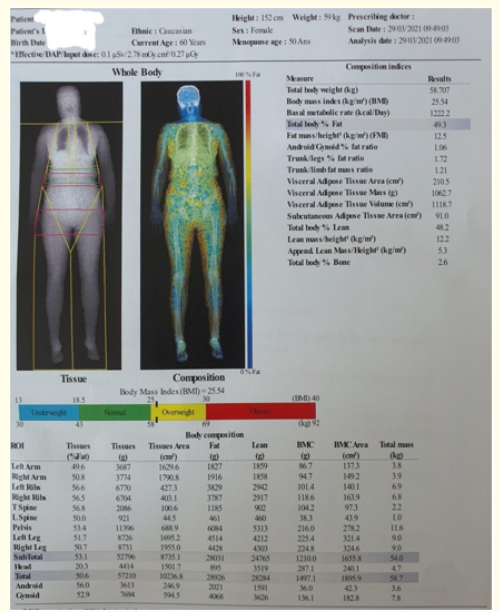

Image 7

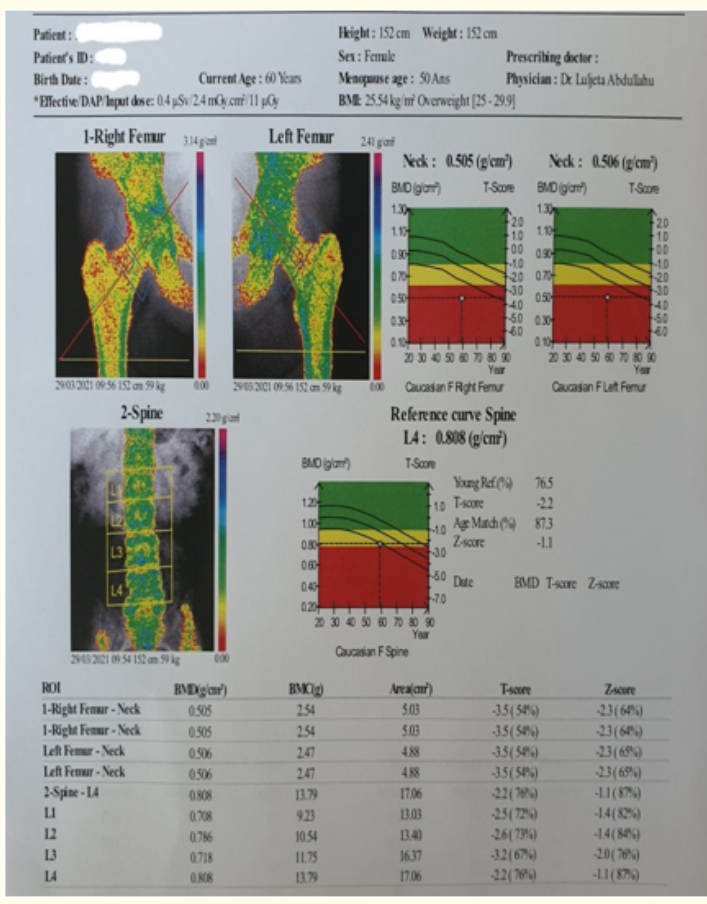

Image 8

As well as the dynamic study for the evaluation of GFR and differential function with 99mTc-DTPA, we found hypofunction to renal insufficiency due to nephrocalcinosis (Image 9).

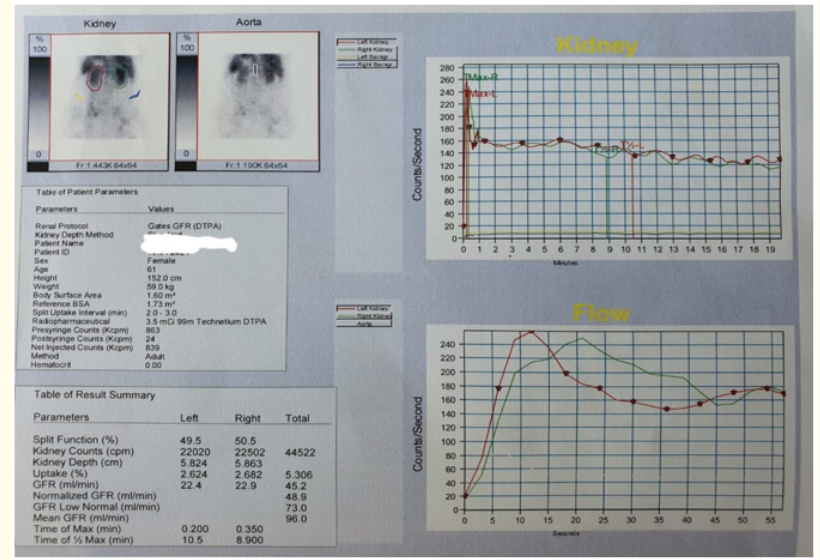

Image 9 
Parathyroidectomy is the only curative treatment for pHPT

- $\quad$ St. Post op. parathyroidectomy

- $\quad$ H.P. parathyroid adenoma.

\section{Conclusion}

The MIBI scan accurately detected and located adenomas in over $90 \%$ of cases. In conclusion, the parathyroid image obtained using a single radionuclide with MIBI (early and late study with differential leaching analysis) is a promising procedure in the preliminary detection and localization of parathyroid adenomas in patients with primary hyperparathyroidism.

Exploration of the neck before invasive surgery, using imaging methods with the $99 \mathrm{mTc}$ agent sestamibi for the detection and localization of parathyroid gland adenoma, helps in focused and successful parathyroidectomy.

A parathyroid scan with MIBI is performed only in a nuclear medicine department of a public hospital, ultrasound in the radiology clinic. We were able to provide this service, supplementing it with additional imaging methods, such as DEXA, DTPA Dynamic Kidney Scintigraphy and Myocardial Perfusion Scintigraphy to verify complications.

\section{Bibliography}

1. Lundgren E., et al. "Population-based screening for primary hyperparathyroidism with serum calcium and parathyroid hormone values in menopausal women". Surgery 121 (1997): 287-294.

2. Levin $\mathrm{KE}$ and Clark $\mathrm{OH}$. "The reasons for failure in parathyroid operations". Archives of Surgery 124 (1989): 911-915.

3. Giordano A., et al. "New trends in parathyroid scintigraphy". European Journal of Nuclear Medicine 28 (2001): 1409-1420.

4. Haber RS., et al. "Ultrasonography for preoperative localization of enlarged parathyroid glands in primary hyperparathyroidism: comparison with $(99 \mathrm{~m})$ technetium sestamibi scintigraphy". Clinical Endocrinology (Oxf) 57 (2002): 241-249.

5. Farley DR. "Technetium-99m-methoxyisobutyl isonitrilescintigraphy: preoperative and intraoperative guidance for primary hyperparathyroidism". World Journal of Surgery 28 (2004):
1207-1211.

6. Bugis SP., et al. "Technetium99m-sestamibi scanning before initial neck exploration in patients with primary hyperparathyroidism". European Archives of Otorhinolaryngology 252.3 (1995): 149-152.

7. Akerström G., et al. "Surgical anatomy of human parathyroid glands". Surgery 95 (1984): 14-21.

8. Al Zahrani AA and Levine MA. "Primary hyperparathyroidism". Lancet 349 (1997): 1233-1238.

9. International Commission on Radiation Protection. ICRP publication 80. Radiation dose to patients from radiopharmaceuticals. Addendum 2 to ICRP Publication. Oxford: Pergamon Press (1998).

10. Administration of Radioactive Substances Advisory Committee. "Notes for guidance on the clinical administration of radiopharmaceuticals and use of sealed radioactive sources". (1998).

11. “Parathyroid Adenoma” (2011).

Volume 5 Issue 9 September 2021

(C) All rights are reserved by Luljeta Abdullahu and Naser Gjonbalaj. 